\title{
Spectral Contents of Electron Waves under Strong Langmuir Turbulence
}

\author{
M. Virgínia Alves ${ }^{1}$, R. S. Dallaqua ${ }^{1}$, F. Do Prado ${ }^{2}$, and D. M. Karfidov ${ }^{3}$ \\ ${ }^{1}$ Instituto Nacional de Pesquisas Espaciais, Laboratório Associado de Plasma \\ P. O. Box 515, 12243-971 - São José dos Campos, SP, Brazil \\ ${ }^{2}$ Centro Universitário da Fundação Educacional Inaciana - UNIFEI \\ Av. Humberto de A.C. Branco, 3972, 09859-901, S. B. do Campo, SP, Brazil \\ ${ }^{3}$ General Physics Institute, Moscow 119991, Russia
}

Received on 10 August, 2002. Revised version received on 28 March, 2003

\begin{abstract}
In this work we present experimental results of electron plasma waves excited in a beam plasma system. Based on our experimental results we determine the transition from the quasi-linear to non-linear regime. We present the space evolution of the electron beam distribution function for both regimes. The spectrum of the electron plasma wave in the non-linear regime shows a component with frequency larger than the plasma frequency besides the plasma frequency itself. We show that the higher frequency component is strongly affected by Landau damping, indicating a dissipation region. The measured experimental power spectrum of this wave shows a dependence on wave number $k$ given by $W_{k} \propto k^{-7 / 2}$ as theoretically predicted.
\end{abstract}

\section{Introduction}

A system formed by a weak non-relativistic electron beam with a unmagnetized collisionless plasma constitutes one of the most interesting configurations for studying plasma wave-particle interactions. The relaxation of the beam provides the free energy source for a rich variety of nonlinear processes. According to quasi-linear (QL) theory the beam relaxation occurs through "plateau" formation by flattening the beam velocity distribution, $f_{b}(v)$, when the beam flows across the plasma[1]. The most unstable electrostatic wave has frequency near the plasma frequency, $\omega_{L} \simeq \omega_{p e}=\left(n_{0} e^{2} /\left(\epsilon_{0} m_{e}\right)\right)^{1 / 2}$, and wave number given by $k_{0} \simeq \omega_{p e} / v_{b}$, where $v_{b}$ is the beam velocity. Depending on the beam energy, the amplitude of excited waves can be high enough to become a pump wave for wave-wave interactions such as modulational instability (MI)[2]. The main nonlinear effect related to $\mathrm{MI}$ is the trapping of waves by density cavities that occurs spontaneously in the plasma as the instability develops. In turn, the trapped waves steep the density even more and the process continues as the waves collapse to smaller and smaller spatial lengths while their amplitude increases. At the collapse burn out stage, when the cavity is small enough for the absorption of Langmuir wave to start, the high-frequency pressure drops quickly and the excess density variations are released as ion-sound waves emitted from the cavity. The evolution of the MI leads the system to the strong Langmuir turbulence (SLT) regime [3-5].

More than twenty years ago a detailed experimental test of quasi-linear theory was performed by Roberson and collaborators[6]. They measured the final state of the beam- plasma instability, and found good agreement with QL theory for the final spectrum and velocity distribution. A few years later, the nonlinear evolution of the electron beamplasma instability started to be investigated [7-10]. The most recent experimental results on strong Langmuir turbulence in the beam plasma interaction have been presented by Wong and collaborators [11-13]. They explore the time evolution of the electrostatic wave spectra, during several ion plasma periods.

In this paper we determine experimental conditions for observing strong Langmuir turbulence in our device. We establish the threshold for the transition between quasi-linear and nonlinear regime as a function of beam to plasma density ratio $\left(n_{b} / n_{0}\right)$ and beam energy normalized to thermal energy $\left(W_{b} / n_{0} T_{e}\right)$. We measure the beam relaxation length and amplitude waves as a function of $W_{b} / n_{0} T_{e}$, for different values of $n_{b} / n_{0}$. We obtain the time growth rates for the excited waves and compare them with existing theory. We show that the excitation of MI leads the system to SLT regime. In this regime, we detect the presence of electron wave with frequency larger than the beam resonant wave. We show that for this higher frequency wave Landau damping acts effectively. The measured experimental power spectrum of this wave shows a dependence on wave number $k$ given by $W_{k} \propto k^{-7 / 2}$ as theoretically predicted [14].

The paper is organized as follows. In Section 2 we describe the experimental apparatus and the diagnostics. In Section 3 we present the experimental conditions for the excitement of non-linear phenomena. We report and examine the excited electron wave in Section 4. Section V presents our conclusions. 


\section{Experimental apparatus and diag- nostics}

Experiments were carried out in a double QUIescent Plasma (PQUI) device with multipole surface magnetic confinement, $0.60 \mathrm{~m}$ of inner diameter and $1.20 \mathrm{~m}$ of total length [15]. The vacuum background attained using a diffusion pump is of the order of $4 \times 10^{-5} \mathrm{~Pa}$. The device is divided by two grids into a source $\left(l_{s}=0.30 \mathrm{~m}\right)$ and a target plasma $\left(l_{t}=0.90 \mathrm{~m}\right)$ electrically insulated. A quiescent plasma is generated independently in both chambers by accelerating primary electrons produced by tungsten hot cathode filaments. An electron beam is created biasing the source chamber negatively with respect to the grounded target chamber. The DC electron beam is $\approx 0.5 \mathrm{~m}$ in diameter with the ratio of beam to electron thermal velocity in the range of $v_{b} / v_{t h} \leq 20$. The experiments were carried out in Argon gas in the filling pressure range of $10^{-2} \leq p \leq 10^{-1} \mathrm{~Pa}$. Fig. 1 shows a schematic diagram of this apparatus.

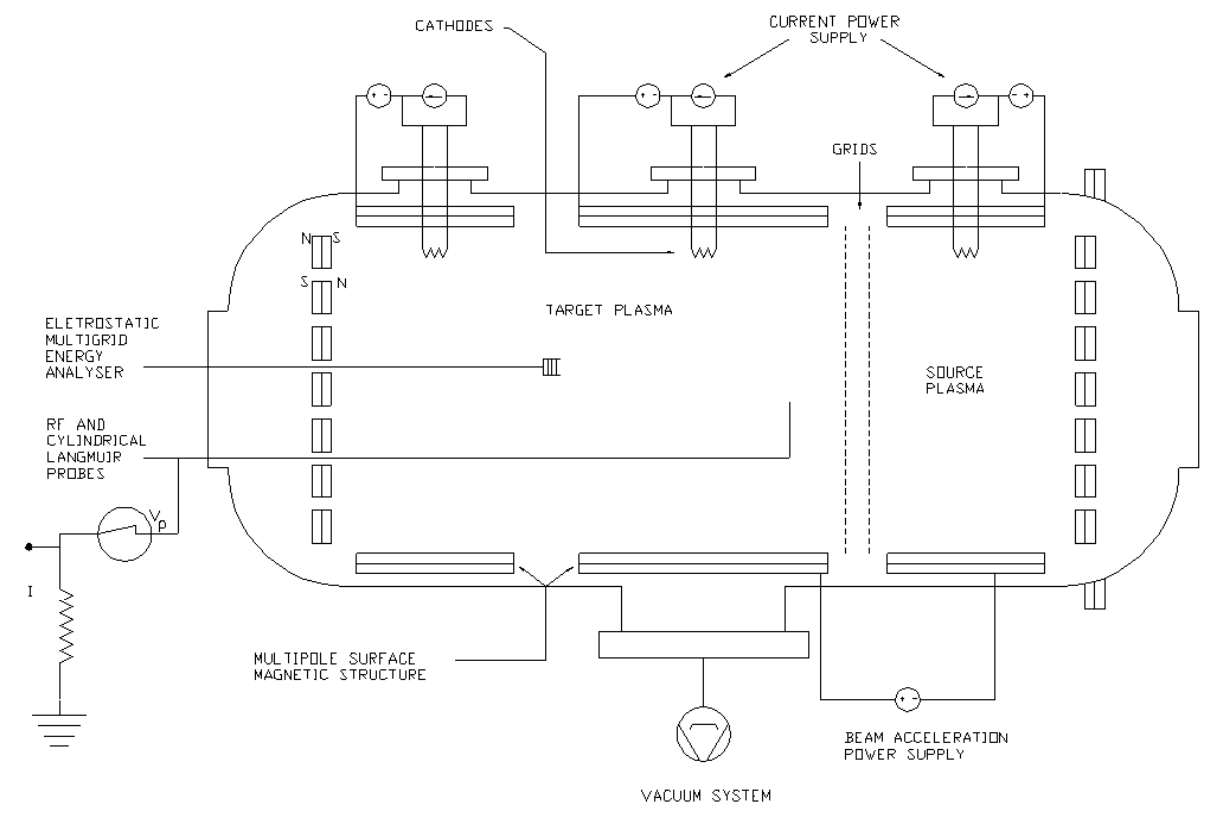

Figure 1. Schematic diagram of the PQUI apparatus.

\begin{tabular}{|ll|}
\hline Table 1 - Typical plasma experimental parameters & \\
Plasma density $\left(\mathrm{m}^{-3}\right)$ & $10^{14}<n_{0}<6.0 \times 10^{15}$ \\
Electron temperature (eV) & $0.7<T_{e}<5$ \\
Electron to ion temperature ratio & $T_{e} / T_{i} \leq 15$ \\
Debye length (m) & $2.1 \times 10^{-4}<\lambda_{D e}<6.2 \times 10^{-4}$ \\
Plasma frequency (MHz) & $90<\omega_{p e} / 2 \pi<690$ \\
Ion plasma frequency (MHz) & $0.33<\omega_{p i} / 2 \pi<2.5$ \\
Beam energy (eV) & $W_{b} \leq 400$ \\
Beam to plasma density ratio & $5 \times 10^{-5}<n_{b} / n_{0}<10^{-2}$ \\
Thermal spread of the beam (at $z \simeq 0 \mathrm{~m})$ & $0.1<(\Delta v) / v_{b}<0.2$ \\
\hline
\end{tabular}

The electron density and temperature measurements were performed using a single cylindrical Langmuir probe. The temperature was determined by the traditional method. The density was obtained by detecting the cut-off frequency of an electromagnetic wave launched into the plasma. The same probe, operating in the ion saturation current regime (probe potential, $V_{p}=-50 \mathrm{~V}$ ), was used for ion-sound oscillation measurements. The typical plasma potential in the target plasma is positive and of the order of 5-10 V. For Langmuir (electron) wave detection a thin wire working as a dipole probe, insulated by a capacitor, is connected to the spectrum analyzer. The evolution of the electron energy distribution function was measured by an electrostatic multigrid energy analyzer using the retarding potential method [16]. Table 1 shows typical plasma parameters for our experiment. 
At the range of working pressure the collisional damping rate due to electron-ion and electron-neutral collisions is smaller than the generation rate of waves, the beam-plasma instability growth rate, $\gamma_{b p}$. This leads to the condition[17]

$$
\pi \frac{n_{b}}{n_{0}}\left(\frac{v_{b}}{\Delta v}\right)^{2}>\frac{\nu_{e n}}{\omega_{p e}}
$$

where $\nu_{e n}$ is the electron neutral collision rate. Once this condition is satisfied, the excitation of Langmuir wave by the beam-plasma instability is allowed. Using the parameters shown in Table 1 we see that Equation 1 is satisfied.

The beam tends to disturb the plasma, so we consider measurements for distances $(z)$ larger than $0.2 \mathrm{~m}$ away from the grid, in the target plasma. At this distance the plasma is uniform in density and temperature, as shown in Fig. 2. The waves are still far from saturation when they enter the uniform region, and all important physics occurs in that region.

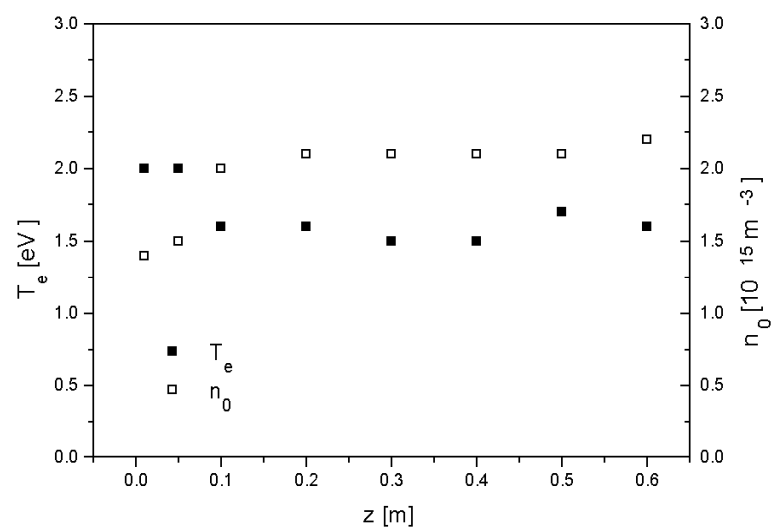

Figure 2. Density and temperature across the PQUI device. The distance $z$ is measured from the injecton grid. Relevant parameters are $W_{b} /\left(n_{0} T_{e}\right)=125$, and $n_{b} / n_{0}=4.1 \times 10^{-4}$.

\section{Transition from QL to SLT regime}

In order to explain the observed waves in our experiment, we need a comprehensive theory. Such theory requires the specification of a free-energy source, a mechanism for wave growth driven by this free-energy source, and a wave energy saturation mechanism. Free energy for the Langmuir wave growth is provided by the beam relaxation. For the range of parameters used in the experiment the Langmuir wave growth is driven primarily by the kinetic version of the beam instability. The saturation mechanism is quasi-linear relaxation or nonlinear processes, depending on the beam plasma parameters $W_{b} / T_{e}$ and $n_{b} / n_{0}[18]$.

From our experimental results it is possible to establish a threshold line for the transition between QL and SLT regimes. We measure the maximum of Langmuir wave amplitude, $W_{L}^{\max }$, as a function of $W_{b} / T_{e}$ for different values of $n_{b} / n_{0}$. Fig. 3 shows the results. Two different behaviours appear: up to a certain value of $W_{b} / T_{e}$, called here $\left(W_{b} / T_{e}\right)_{t h}$, the best fit of the experimental points gives $W_{L}^{\max } \propto\left(W_{b} / T_{e}\right)^{2.0 \pm 0.3}$, independent of the value of $n_{b} / n_{0}$. For $W_{b} / T_{e}>\left(W_{b} / T_{e}\right)_{t h}$, the wave amplitude remains approximately constant. Fig. 3 also shows that $W_{L}^{\max }$ increases with $n_{b} / n_{0}$.

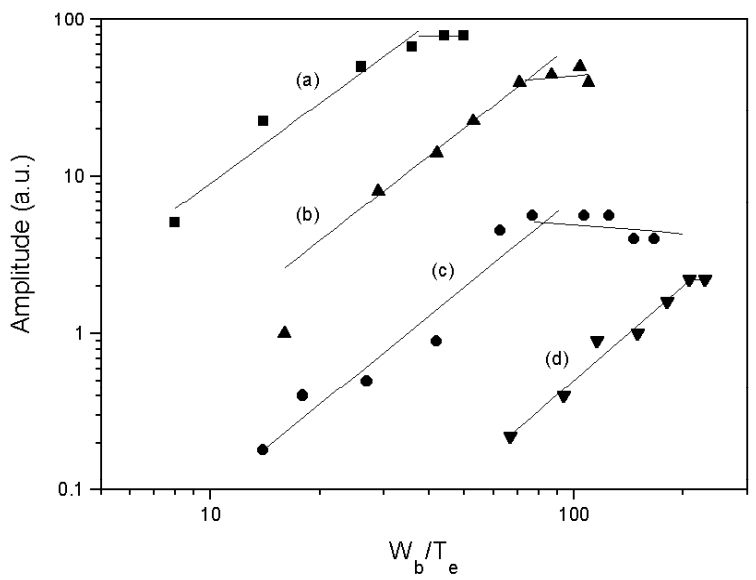

Figure 3. Maximum of electron wave amplitude as a function of $W_{b} / T_{e}$ for different values of $n_{b} / n_{0}$ : (a) $4.4 \times 10^{-3}$, (b) $1.7 \times 10^{-3}$, (c) $4.1 \times 10^{-4}$, and (d) $1.3 \times 10^{-4}$. Lines are the best fit to the experimental points, considering different ranges of beam energy.

According to quasi-linear theory, and considering pile up effects, the maximum normalized energy density of the waves is given by[18]

$$
\overline{W_{L}^{\max }}=\frac{\epsilon_{0}\left|E_{0}\right|^{2}}{2 n_{0} T_{e}}=\frac{1}{18} \frac{n_{b}}{n_{0}}\left(\frac{W_{b}}{T_{e}}\right)^{2} .
$$

Therefore, for $W_{b} / T_{e}<\left(W_{b} / T_{e}\right)_{t h}$, our experimental results show the same dependence of $W_{b} / T_{e}$ and $n_{b} / n_{0}$ as predicted by QL theory.

Stabilization of maximum wave amplitude suggests the appearance of a nonlinear phenomenon that interferes with the beam-plasma instability evolution. Together with the saturation of wave energy we see the appearance of ionsound waves[19].

From Fig. 3, for a given value of $n_{b} / n_{0}$, we obtain the value of $\left(W_{b} / T_{e}\right)_{t h}$. These points are shown in Fig. 4, identified by circles, together with the points where ion sound waves are observed, identified by triangles. The line shown in Fig. 4 is the theoretical threshold for MI excitement in an electron beam plasma system [20]

$$
\left(\frac{n_{b}}{n_{0}}\right)_{t h} \simeq\left(\frac{T_{e}}{W_{b}}\right)_{t h}^{3}
$$




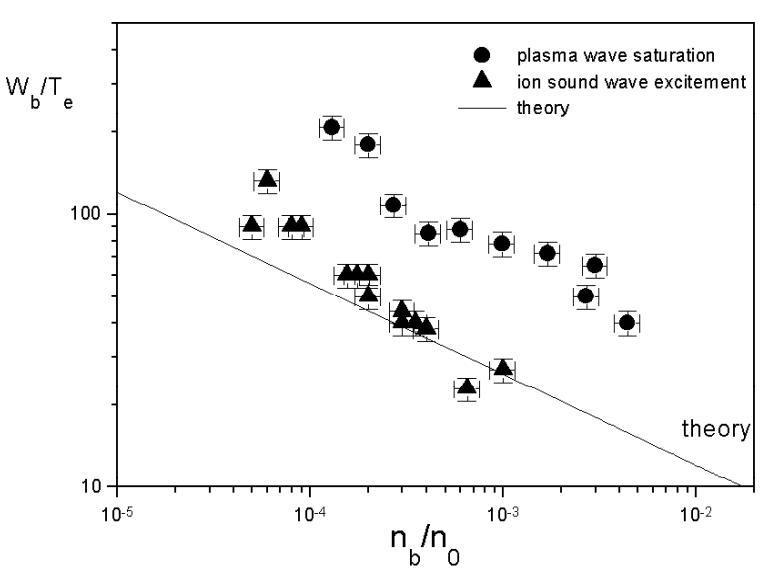

Figure 4. Threshold conditions for transition from quasi-linear electron beam-plasma interaction to strong turbulence regime in a plane of parameters $W_{b} / T_{e}$ and $n_{b} / n_{0}$.

For a system with parameters in the region below the MI excitation threshold, the Langmuir wave energy saturates due to the complete relaxation of the beam. The position of saturation of the wave energy corresponds to the position of plateau formation in the distribution function, as predicted by quasi-linear theory (known as "beam relaxation length"). For a system with parameters in the region above the MI excitement threshold, the nonlinear instability transports the plasma waves to shorter wavelength scales (higher frequencies) where the resonant interaction between beam and waves does not occur. As a consequence the beam propagates for larger distances than the ones predicted by QL theory.

The axial evolution of the electron beam distribution function shows clearly the different behaviour for the quasilinear and for the nonlinear regime, as we can observe in Fig. 5. Figs. 5(a) and (b) show the quasi-linear case, with $W_{b} / T_{e}=42$, and Figs. 5(c) and (d) show the nonlinear case, with $W_{b} / T_{e}=125$. For both cases $n_{b} / n_{0}=$ $4.1 \times 10^{-4}$. Figs. 5(a) and (c) show the electron beam distribution function for two different axial positions. Figs. 5(b) and (d) show the wave amplitude normalized by the maximum wave amplitude and the spread of the beam $\Delta v / v_{b}$ against position. These figures show that the maximum of the wave amplitude coincides with the complete beam relaxation $\left(\Delta v / v_{b}=1\right)$ for the quasi-linear case. For the nonlinear case, even for $z=0.5 \mathrm{~m}$ it is possible to identify a beam. The values of $\Delta v / v_{b}$ were obtained using a Gaussian fit to the data as shown in Figs. 5(a) and (c).
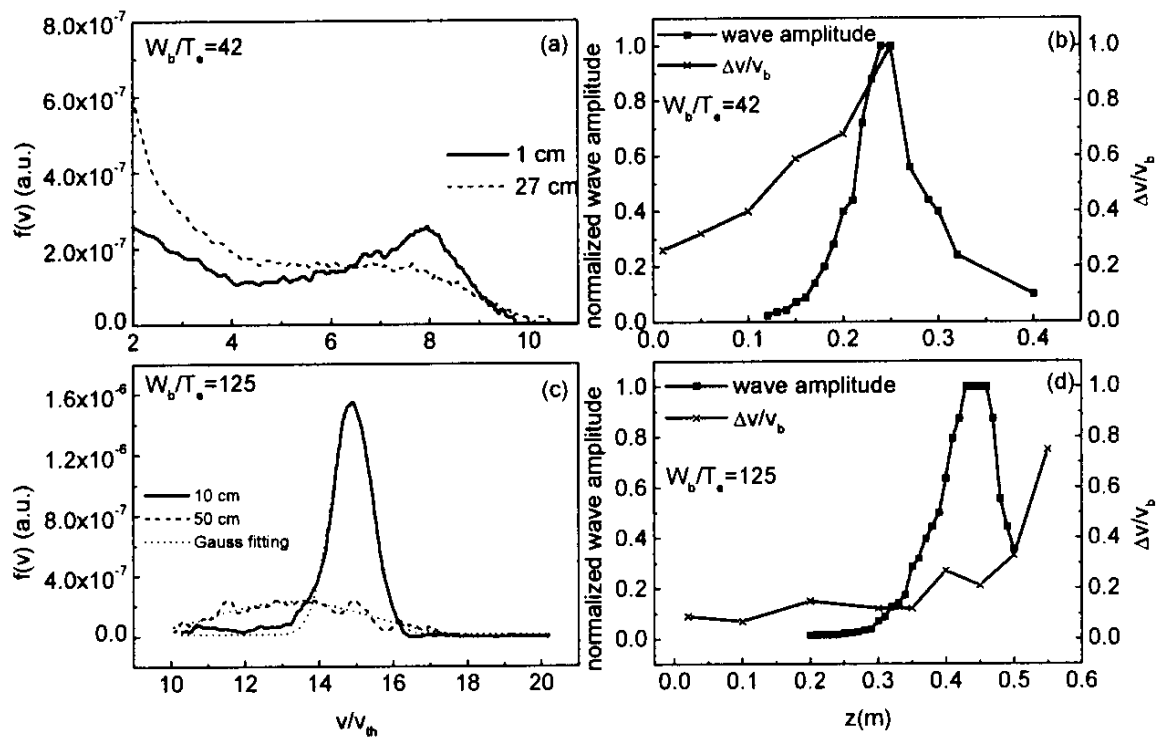

Figure 5. Electron beam distribution function at two different positions: (a) for the quasi-linear case, $W_{b} / T_{e}=42$ and (c) for the nonlinear case, $W_{b} / T_{e}=125$. For both cases $n_{b} / n_{0}=4.1 \times 10^{-4}$. Axial variation of the normalized wave amplitude and the velocity beam spread: (b) for the quasi-linear case and (d) for the nonlinear case.

Figure 6 shows the normalized beam relaxation length, $L_{n}=l \omega_{p e} / v_{b}$, as a function of $W_{b} / T_{e}$ for three different values of $n_{b} / n_{0}$. The length $l$ is the distance between the grid and the axial position where the plateau is formed in the distribution function. The QL theory gives the beam relaxation length as $L_{n}=\left(n_{b} / n_{0}\right)^{-1}\left(W_{b} / T_{e}\right)^{-1} \Lambda$, where $\Lambda$ is of order of the Coulomb logarithm[20]. The lines shown in Fig. 6 are obtained using $\Lambda=8$ and the corresponding $n_{b} / n_{0}$. Experimental results show that for $W_{b} / T_{e}>100$, measured beam relaxation lengths are larger than the ones predicted by QL theory.

To illustrate the evolution of the excited Langmuir waves across the system we show the spectra of the electron waves in Fig. 7. The relevant parameters are $W_{b} / T_{e}=125$, $n_{b} / n_{0}=4.2 \times 10^{-4}$, and $n_{0}=1.8 \times 10^{15} \mathrm{~m}^{-3}$, above the threshold line in Fig. 4. Plots of the spectrum were obtained in three different axial positions. The first spectrum (A) was obtained at $z=0.30 \mathrm{~m}$, and presents a single peak 
at $f_{L}=380 \mathrm{MHz}$, close to the plasma frequency (beam resonant wave). The second spectrum (B) was obtained at $z=0.40 \mathrm{~m}$ and presents the main peak, $f_{L}=380 \mathrm{MHz}$, followed by the second harmonic, $f \sim 740 \mathrm{MHz}$. Harmonics of the main frequency have already been observed[21]. The third plot was obtained at $z=0.50 \mathrm{~m}$ and shows a broader peak at $f_{L}=380 \mathrm{MHz}$, indicating the presence of waves with frequency larger than the frequency of the beam resonant wave. The second harmonic is still present with smaller amplitude than in case B. The wave amplitude increases with the distance from the grid and reaches a maximum value at $z \simeq 0.4 \mathrm{~m}$ and then decreases for larger distances. The space evolution of the Langmuir wave amplitude determines the behaviour of the system.

\section{Analysis of the electron waves in the nonlinear regime}

In this section we concentrate on the electron waves observed in the nonlinear regime. The results obtained from the experimental date are compared with the existing theory.

It is important to point out that we have a continuous beam injection. This fact leads us to a situation where close to the grid, at the point of beam injection, we expect that the excited wave amplitude grows as predicted by QL theory. For larger distances, the behaviour of the system depends on the wave energy density, $W_{L}=\epsilon_{0}\left|E_{0}\right|^{2} / 2$. After reaching a certain threshold, the excited wave can became a pump wave for wave-wave interactions. Starting from the space profile along $z$ of the wave amplitude we obtain the spatial growth rate, $\kappa$, for the instability and from that we obtain the temporal growth rate, $\gamma$. To obtain the results we present in this section we have used $n_{0}=2.1 \times 10^{15} \mathrm{~m}^{-3}$, $n_{b} / n_{0}=4.1 \times 10^{-4}$ and $W_{b} / T_{e}=125\left(v_{b} \sim 16 v_{t h}\right)$. Notice that with these parameters we are above the threshold MI line shown in Fig. 4, i.e., the system operates in the SLT regime.

The axial profiles of the electron wave amplitude at frequencies, $f_{L}=410 \mathrm{MHz}$, close to the plasma frequency, and at $f^{*}=490 \mathrm{MHz}$, are shown in Fig. 8 (curves (a) and (b), respectively). Observe that the higher frequency wave only appears after the Langmuir wave has reached region labeled by 2 .

Notice that from our experimental results we can only obtain the spatial growth rate. In general, beam-plasma instabilities exhibit both temporal and spatial growth. A simple relationship between them is given when the beam can be considered cold $(c)$ or hot $(h)$, as regards both temporal and spatial growth[22].

The experimental values of the spatial growth rate can be obtained from Fig. 8 as follows. The spatial growth rate is $\kappa_{e x}=1 / \zeta$, where $\zeta$ is the distance over which the wave amplitude grows by a factor of $e \simeq 2.7$. Within region labeled by 1 in Fig. 8 we obtain $\kappa_{1 e x} / k_{0}=0.11$ and within region labeled by 2 we obtain $\kappa_{2 e x} / k_{0}=0.04$.

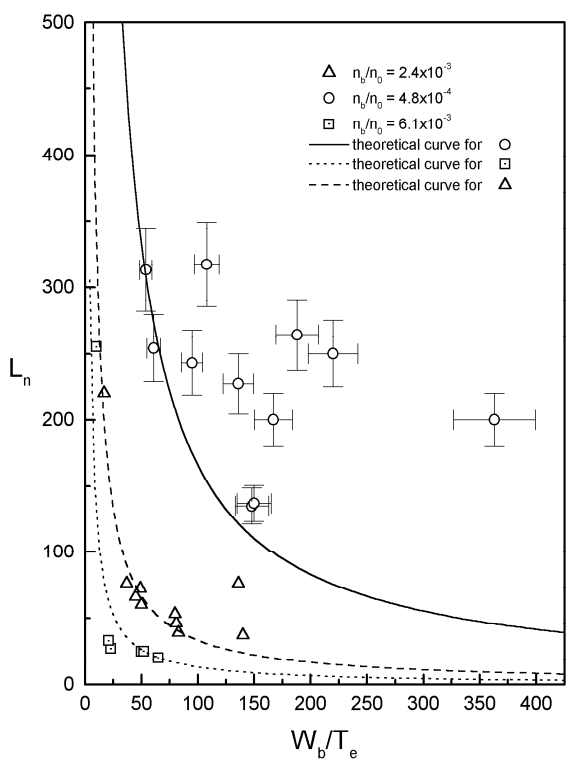

Figure 6. Measured (symbols) and theoretical (QL) relaxation length (lines) against $W_{b} / T_{e}$, for $n_{b} / n_{0}=4.8 \times 10^{-4}, 2.4 \times 10^{-3}$, and $6.1 \times 10^{-3}$.
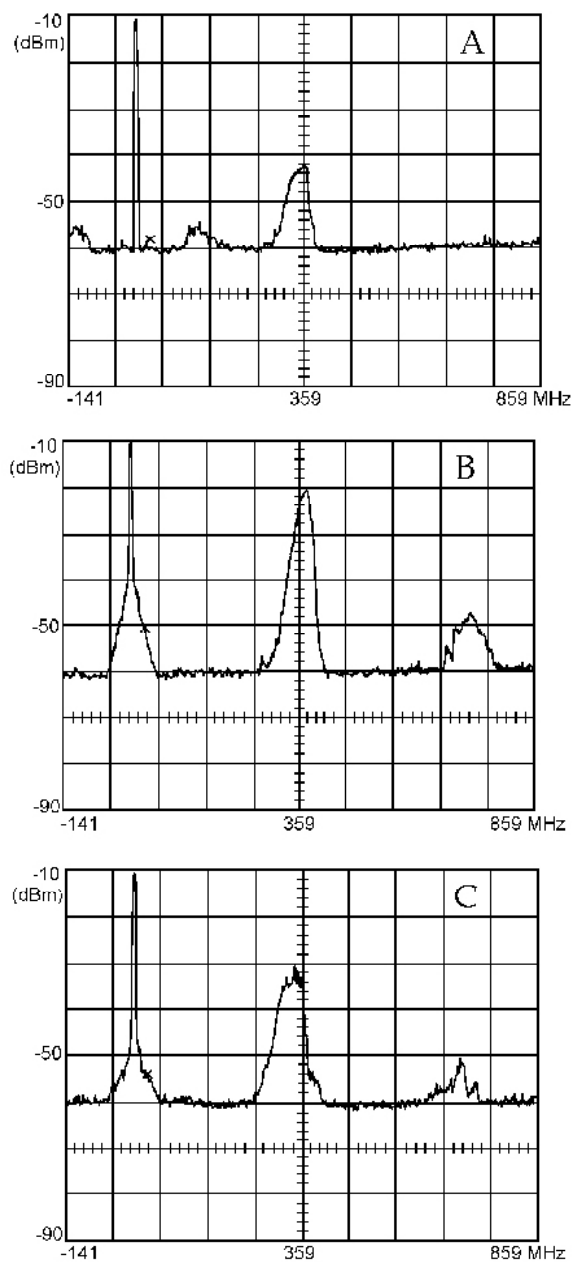

Figure 7. Frequency spectra of Langmuir waves obtained in different positions in the target chamber: A for $z=0.3 \mathrm{~m}$, B for $z=0.4$ $\mathrm{m}$, and $\mathrm{C}$ for $z=0.5 \mathrm{~m}$. 
The theoretical spatial growth rates are given by[22]

$$
\begin{gathered}
\frac{\kappa_{c}}{k_{0}}=\left(\frac{3^{1 / 2}}{2}\right)\left(\frac{n_{b}}{6 n_{0}}\right)^{1 / 3}\left(\frac{v_{b}}{v_{t h}}\right)^{2 / 3} \\
\frac{\kappa_{h}}{k_{0}}=\left(\frac{\pi}{2 e}\right)^{1 / 2}\left(\frac{n_{b}}{6 n_{0}}\right)\left(\frac{v_{b}}{v_{t h}}\right)^{2}\left(\frac{v_{b}}{\Delta v_{b}}\right)^{2} .
\end{gathered}
$$

Within region 1 we have $n_{b} / n_{0}=4.1 \times 10^{-4}$, $\Delta v_{b} / v_{b}=0.1$ and $v_{b}=16 v_{t h}$ which gives $\Delta v_{b} / v_{b}<$ $\left(n_{b} / 6 n_{0}\right)^{1 / 3}\left(v_{b} / v_{t h}\right)^{2 / 3}<1$. The beam can be considered cold as regards spatial growth rate and Equation (4) gives $\kappa_{c 1} / k_{0}=0.22$, the same order of magnitude as $\kappa_{1 e x} / k_{0}$.

The theoretical temporal growth rates are given by[22]:

$$
\begin{gathered}
\frac{\gamma_{c}}{\omega_{p e}}=3^{1 / 3}\left(\frac{v_{t h}}{v_{b}}\right)^{2 / 3}\left(\frac{\kappa_{c}}{k_{0}}\right)=\left(\frac{3^{1 / 2}}{2}\right)\left(\frac{n_{b}}{2 n_{0}}\right)^{1 / 3} \\
\frac{\gamma_{h}}{\omega_{p e}}=3\left(\frac{v_{t h}}{v_{b}}\right)^{2}\left(\frac{\kappa_{h}}{k_{0}}\right)=\left(\frac{\pi}{2 e}\right)^{1 / 2}\left(\frac{n_{b}}{2 n_{0}}\right)\left(\frac{v_{b}}{\Delta v_{b}}\right)^{2} .
\end{gathered}
$$

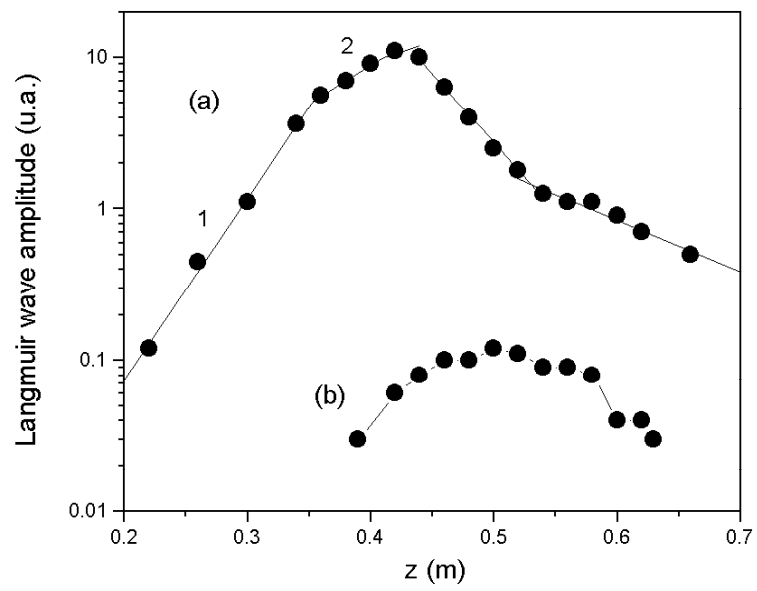

Figure 8. Axial profiles of the high frequency electric field (Langmuir waves) at two different frequencies, for $n_{b} / n_{0}=4.1 \times 10^{-4}$ and $W_{b} / T_{e}=125$ : (a) Langmuir wave frequency, $410 \mathrm{MHz}$ and (b) high frequency, $490 \mathrm{MHz}$.

As regards temporal growth the beam can be considered marginally hot, since $\left(n_{b} / 6 n_{0}\right)^{1 / 3}\left(v_{b} / v_{t h}\right)^{2 / 3}<$ $\Delta v_{b} / v_{b}<1$. For the experimental parameters we obtain $\gamma_{1 c} / \omega_{p e}=5 \times 10^{-2}$ and $\gamma_{1 h} / \omega_{p e}=1.5 \times 10^{-2}$. Since the beam is only marginally hot let us assume that we can consider the beam cold as regards both temporal and spatial growth and use Equation (6) to obtain the temporal growth rate related to $\kappa_{1 e x}$. In that case $\gamma_{c 1 e x} / \omega_{p e}=2.3 \times 10^{-2}$, a value in between the values obtained previously, and we can say that in region 1 the beam-plasma is the dominant instability, and the system behaves as predicted by QL theory.

Within region $2, \Delta v_{b} / v_{b} \simeq 0.25$ (see Fig. 5), and the beam can be considered hot as regards both temporal and spatial growth rates. In that case the temporal growth rate $\gamma_{2 e x}$ can be estimated through the relation $\gamma=\kappa v_{g}$, where $\kappa$ is the spatial growth rate and $v_{g}=3 v_{t e}^{2} / v_{b}$ is the group velocity of Langmuir waves[22]. These considerations give $\gamma_{2 e x} / \omega_{p e}=4.7 \times 10^{-4}$.
Within the region labeled by 2 the time growth rate $\gamma$ of the main frequency $f_{L}=410 \mathrm{MHz}$ decreases due to excitation of modulational instability (MI). MI creates an effective dissipation mechanism of electron waves. This process results in a corresponding decreasing of QL growth rate according to expression $\gamma_{2}=\gamma_{b p}-\nu_{e f f}$, where $\nu_{e f f}$ is the effective collisional frequency of turbulence, characterizing the rate of transference of energy wave into the short wavelength region of the spectrum. From $\gamma_{2 e x}=\gamma_{2 h}-v_{\text {eff }}$ we obtain $v_{\text {eff }} / \omega_{p e} \simeq 2.1 \times 10^{-3}$.

Curve (b) of Fig. 8 gives $\kappa_{2 e x}^{*} / k_{0}=0.06$. Assuming that the excited wave, $f^{*}$, follows the Bohm-Gross dispersion relation and using $\gamma=\kappa v_{g}$ we obtain $\gamma_{e x}^{*} / \omega_{p e}=$ $1.7 \times 10^{-3}$. Assuming that the appearance of the high frequency wave at $f^{*}=490 \mathrm{MHz}$ in region 2 is due to the MI, we could say that $\gamma_{e x}^{*} / \omega_{p e}=\gamma_{M I} / \omega_{p e} \approx 1.7 \times 10^{-3}$, which is very close to the value obtained for $\nu_{\text {eff }} / \omega_{p e}$.

The relevant growth rate of modulational instability can be estimated by[20]

$$
\frac{\gamma_{M I}}{\omega_{p e}}=\left(\frac{m_{e} W_{L}}{3 m_{i} n_{o} T_{e}}\right)^{\frac{1}{2}}
$$

where $W_{L}$ is the pump Langmuir wave amplitude.

We have not measured directly the value of $W_{L}$. However, the value of $W_{L}$ can be estimated in different ways. Using Equation 2 we obtain $\overline{W_{L}}=W_{L} / n_{0} T_{e}=0.36$. On the other hand, considering energy balance condition, $\gamma_{b p} \simeq \gamma_{M I}$, we obtain $\overline{W_{L}} \approx 0.32$. The maximum of energy of Langmuir wave, resonant with the electron beam, can also be attained from the normalized relaxation length of the beam[24]

$$
\overline{W_{L}} \simeq \frac{1}{L_{n}} \frac{W_{b}}{T_{e}}
$$

For the experimental conditions described before we have $L_{n} \simeq 250$ (see Fig. 6), which gives $\overline{W_{L}} \simeq 0.5$.

Another estimative for $\overline{W_{L}}$ is provided by numerical simulation of the system. Simulations were performed using 
the electrostatic code XPD1[23], incorporating three species of charged particles: background electrons and ions with maxwellian distribution functions and beam electrons with a drifting maxwellian distribution function. We choose as initial conditions the following parameters, typical for our experimental system in the nonlinear regime: $n_{0}=2 \times 10^{15}$ $\mathrm{m}^{-3}, T_{e}=2 \mathrm{eV}, T_{e} / T_{i}=10, v_{b}=9.6 \times 10^{6} \mathrm{~m} / \mathrm{s} \simeq 16 v_{t e}$, $\Delta v / v_{b}=0.1, m_{e} / m_{i}=1 /(40 \times 1836), n_{b} / n_{0}=5 \times 10^{-4}$, and $W_{b} / T_{e}=125$. Fig. 9 shows the electric field across the system obtained from simulations for a time $t \approx 18 \omega_{p i}^{-1}$. For $z \simeq 0.4 \mathrm{~m}$ we obtain $E_{0} \simeq 5 \mathrm{kV} / \mathrm{m}$, which gives $\overline{W_{L}} \simeq 0.2$.

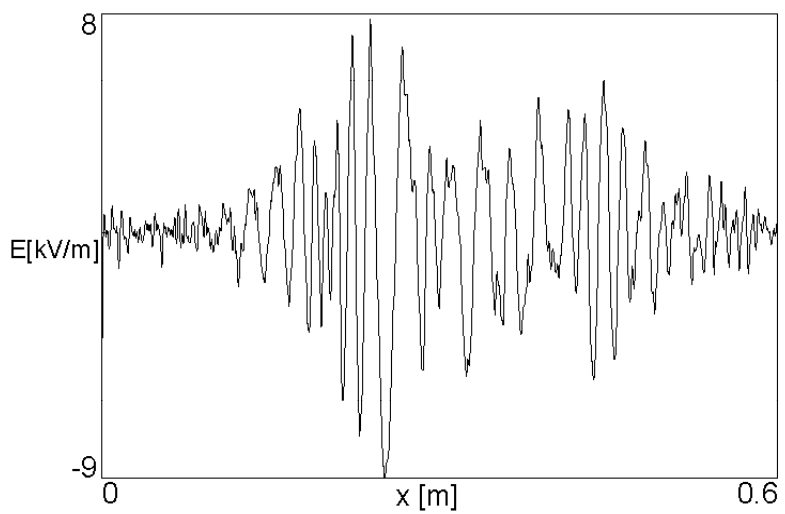

Figure 9. Axial profile of the electric field obtaind from simulations for a time $t \simeq 18 \omega_{p i}^{-1}$.

In summary, either way we reckon the value of $\overline{W_{L}}$, we obtain $0.2<\overline{W_{L}} \simeq 0.5$, and the MI growth rate given by Equation 8 stays in the range $1 \times 10^{-3}$ up to $1.5 \times 10^{-3}$, in good agreement with both $\nu_{e f f} / \omega_{p e}$ and $\gamma_{e x}^{*} / \omega_{p e}$. Also $\overline{W_{L}}>>\left(k \lambda_{D}\right)^{2}$, a condition to strong Langmuir turbulence.

The values of wave number involved in these processes can be obtained as follows. Using the Bohm-Gross dispersion relation for small values of $k \lambda_{D e}$

$$
\left(\frac{f}{f_{p e}}\right) \simeq 1+\frac{3}{2}\left(k \lambda_{D e}\right)^{2}
$$

we obtain $k \lambda_{D e} \sim 0.36$ for the wave with $f^{*}=490 \mathrm{MHz}$, and $f_{p e} \simeq 412 \mathrm{MHz}$. The absorption of the highest frequency component is an important characteristic at the final stage of the SLT regime. The plasma bulk electrons are accelerated by Landau damping. Theoretical expression for Landau damping is

$$
\frac{\Gamma_{k}}{\omega_{p e}}=\frac{\pi}{8} \frac{1}{\left(k \lambda_{D e}\right)^{3}} \exp \left(-1 /\left(2 k^{2} \lambda_{D e}^{2}\right)-3 / 2\right) .
$$

For $k \lambda_{D e} \sim 0.36$, Equation 11 gives $\Gamma_{k} / \omega_{p e}=4.0 \times 10^{-2}$. We see then that for the region of the spectra $k \lambda_{D e} \sim 0.36$, Landau damping acts effectively, compared to the others growth rates previously calculated. According to theory, the turbulent energy concentrated in the high frequency waves is absorbed by plasma electrons, leading to acceleration of bulk electrons, as experimentally observed[19]. The theoretical results on power spectra for this absorption is given by $W(k) \propto k^{-7 / 2}[14]$. Fig. 10 shows the experimental results for the power spectra presenting a good agreement with the theory.

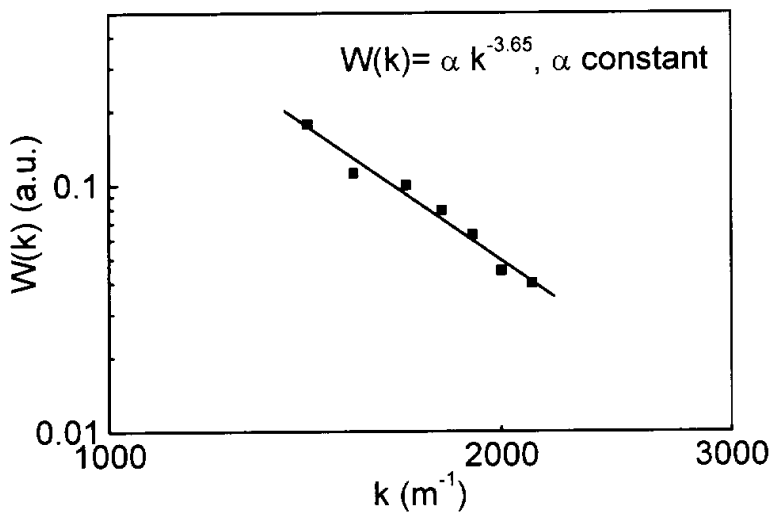

Figure 10. Power spectra of the higher frequency electron plasma wave.

\section{Conclusions}

We present experimental measurements of electron waves excited in a system operating in a Langmuir turbulence regime. The interaction of a non-relativistic warm electron beam with a unmagnetized plasma drives the turbulence. We establish experimental threshold conditions for transition from the QL into the SLT regime as a function of beam and plasma parameters, $W_{b} / T_{e}$ and $n_{b} / n_{0}$. The operating regime determines the appearance and characteristics of Langmuir waves. At regions close to the grid, point of the injection of the beam, the Langmuir wave grows according to QL theory. As the wave amplitude reaches a certain threshold it becomes a pump wave for exciting other waves. A wave with frequency larger than the frequency of the beam resonant wave appears. Analysis of the growth rates indicate that this wave is a consequence of the development of the modulational instability. It was shown that this component is strongly affected by Landau damping and the measured power spectra is in good agreement with the theory. Analysis of the effects of the ion waves on the dynamics on the Langmuir turbulence will be addressed in a near future.

\section{Acknowledgments}

The authors thank the Brazilian Agency CNPq (Conselho Nacional de Desenvolvimento Científico e Tecnológico) for partially supporting this work.

\section{References}

[1] D. B. Melrose, Instabilities in space and laboratory plasmas, Cambridge University, pp.45-50 (1986).

[2] P. A. Robinson, Reviews of Modern Physics, 69, 507 (1997). 
[3] L. N. Degtyarev, R. Z. Sagdeev, G. I. Solovév, V. D. Shapiro and V. I. Shevchenko, Sov. Phys. Plasma Phys., 6, 263 (1980).

[4] V. D. Shapiro and V. I. Shevchenko, in Handbook of Plasma Physics vol. 2, eds. M Rosenbluth and R Sagdeev, p.124, Amsterdam: Elsevier Publ., (1984).

[5] V. E. Zakharov, Sov. Phys. JETP, 35, 908 (1972).

[6] C. Roberson, K. W. Gentle, and P. Nielsen, Phys. Review Lett., 26, 226 (1971).

[7] B. H. Quon, A. Y. Wong, and B. H. Ripin, Phys. Review Lett., 32, 406 (1974).

[8] A. Y. Wong and B. H. Quon, Phys. Review Lett., 34, 1499 (1975).

[9] H. Ikezi, R. P. H. Chang, and R. A. Stern, Phys. Review Lett., 36, 047 (1976)

[10] A. Y. Wong and P. Y. Cheung, Phys. Review Lett., 52, 1222 (1984).

[11] M. D. McFarland and A. Y. Wong, Phys. Plasmas, 4, 945 (1997).

[12] M. D. McFarland and A. Y. Wong, Phys. Plasmas, 8, 110 (2001).

[13] M. D. McFarland and A. Y. Wong, Physical Review Letters, 84, 666 (2000).

[14] A. A. Galeev, R. Z. Sagdeev, Yu. S. Sigov, V. D. Shapiro, and V. I. Shevchenko, Sov. J. Plasma Phys., 1, 5 (1975).
[15] J. L. Ferreira, F. do Prado, M.V. Alves, E. D. Campos, and D. M. Karfidov, Revista de Física Aplicada e Instrumentação, 9, 47 (1994).

[16] F. do Prado, Estudo Experimental de Ondas e Turbulência de Langmuir, PhD Thesis, INPE-6819-TDI, 1997.

[17] D. L. Matthews, M. Pongratz, and K. Papadopoulos, J. Geophys. Research, 81, 123 (1976).

[18] V. N. Tsytovich, Nonlinear effects in plasma, New York: Plenum Press (1970).

[19] F. Do Prado, D. M. Karfidov, M. V. Alves, and R. S. Dallaqua, Physics Letters A, 248, 86 (1998).

[20] A. A. Gallev, R. Z. Sagdeev, V. D. Shapiro, and V. I. Shevchenko, Sov. Phys. JETP, 45, 266 (1977).

[21] P. Y. Cheung, A. Y. Wong, C. B. Darrow, and S. J. Qian, Phys. Review Lett., 48, 1348 (1982).

[22] S. A.Self, M. M. Shoucri, and F. W. Crawford, J. Applied Phys., 42, 704 (1971).

[23] J. P. Verboncoeur, M. V. Alves, V. Vahedi, and C. K. Birdsall, J. Comp. Physics, 104, 321 (1993).

[24] D. M. Karfidov and N. A. Lukina, Physics Letters A, 232, 443 (1997). 